AIAA 2000-5177

\title{
ADVANCED CONCEPTS, TECHNOLOGIES, AND FLIGHT EXPERIMENTS FOR NASA'S EARTH SCIENCE ENTERPRISE
}

\author{
By \\ Barry D. Meredith \\ NASA Langley Research Center \\ Hampton, Virginia
}

\begin{abstract}
Over the last 25 years, NASA Langley Research Center (LaRC) has established a tradition of excellence in scientific research and leading-edge system developments, which have contributed to improved scientific understanding of our Earth system. Specifically, LaRC advances knowledge of atmospheric processes to enable proactive climate prediction and, in that role, develops first-of-a-kind atmospheric sensing capabilities that permit a variety of new measurements to be made within a constrained enterprise budget. These advances are enabled by the timely development and infusion of new, state-of-the-art (SOA), active and passive instrument and sensor technologies. In addition, LaRC's center-of-excellence in structures and materials is being applied to the technological challenges of reducing measurement system size, mass, and cost through the development and use of spacedurable materials; lightweight, multi-functional structures; and large deployable/inflatable structures. NASA Langley is engaged in advancing these technologies across the full range of readiness levels from concept, to components, to prototypes, to flight experiments, and on to actual science mission infusion. The purpose of this paper is to describe current activities and capabilities, recent achievements, and future plans of the integrated science, engineering, and technology team at Langley Research Center who are working to enable the future of NASA's Earth Science Enterprise.
\end{abstract}

Copyright $\Theta 2000$ by the American Institute of Aeronautics and Astronautics, Inc. No copyright is asserted in the United States under Title 17, U.S. Code. The U.S. Government has a royalty-free license to exercise all rights under the copyright claimed herein for Governmental Purposes. All other rights are reserved by the copyright owner.

\section{ATMOSPHERIC SCIENCE MISSION}

The Langley mission in atmospheric science is to provide understanding of key atmospheric processes and trends through a portfolio of space, aircraft, and ground-based measurements and atmospheric modeling, analysis, and prediction. Langley's leadership role in atmospheric science for ESE has been achieved primarily by proactively identifying emerging issues, developing new measurement capabilities, conducting space-based measurements, developing predictive models, and leading international field missions.

Specific areas of LaRC scientific research are: Water Cycle, Clouds, and Aerosols which includes radiation budget and cloud modeling; tropospheric chemistry addressing pollution/greenhouse gases sources, sinks, and transport; middle atmosphere science which includes energy balance, ozone depletion, and chemistry/dynamics; and Aviation Impacts such as exhaust effects. Langley scientists have recorded significant scientific achievements in each of these areas of research. An example is the development of a comprehensive Earth radiation budget data set, including first cloud radiative forcing measurements. In addition, under the leadership of LaRC atmospheric scientists, the impact of anthropogenic emissions on global atmospheric chemistry and effectiveness of Montreal Protocol were verified.

The strength of the Langley approach to its atmospheric science mission is the integration of scientists, engineers, and technologists into productive teams. From this collaborative teaming approach, which also includes partnerships with industry, small business, universities, other NASA centers, and other government agencies, has come pioneering measurement capabilities. Examples include lidar measurements for high vertical resolution atmospheric 
aerosols and chemistry studies (LITE. LASE) and solar occultation (SAM, SAGE. HALOE) and limb emission measurements (LIMS) for studies of stritospheric chemistry. In fact, a significant portion of all NASA spaceflight atmospheric research instruments has been developed at Langley as shown in figure 1.

These developments have relied on a robust research and technology development program at the center, which has successfully matured atmospheric sensing technologies from concept to measurement system infusion.

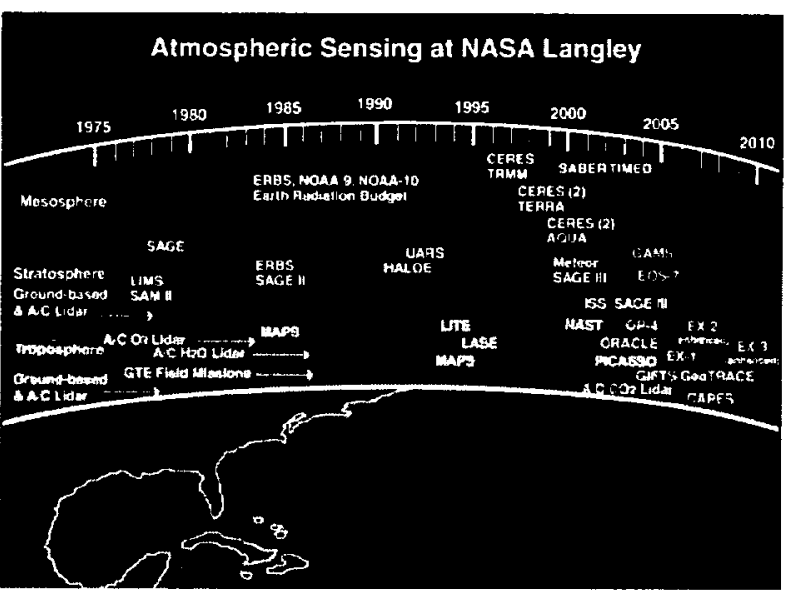

Figure 1. Atmospheric sensing at NASA Langley.

\section{RESEARCH AND TECHNOLOGY}

The process for formulating the technology development program at LaRC features teams of scientists, engineers, and technologists who merge science-driven measurement requirements with advanced concepts and technology opportunities to develop proposed research and technology development projects. These proposals must have direct linkage to the ESE Strategic Plan and the Science Implementation Plan and accommodate technology requirements found in the ESE Capability Needs Database. ISO 9000 procedures define an end-to-end proposal development process, which includes quality reviews, management endorsement, and a "lessons learned" feedback loop for continuous improvement. The finished proposals are then submitted in response to NASA Research Announcements (NRA's) issued by programs that span the range of technology readiness such as the ESE Advanced Technology Initiative (components), Instrument Incubator Program (systems and subsystems), and the New Millennium Program (protoflights for space validation)
There are two primary technology product areas at Langley which support the Earth Science Enterprise. The instrument and sensor area features lasers/lidar technologies as well as passive electro-optic and microwave sensors. Langley is known for its unique, world-class, end-to-end capability in lidar technology and systems. The capability exists to address all phases of development from the invention of new laser sources to the development and deployment of a lidar science instrument either in the field, in an aircraft or in space. LaRC scientists and engineers were the first to demonstrate an atmospheric quality lidar from space via the Shuttle-based LITE mission in 1994. Another first was the successful deployment of a fully autonomous, differential absorption lidar in a high altitude ER-2 aircraft. The Langley R\&T program has also enabled new, more precise measurements of the Earth's troposphere and stratosphere through the development of advanced radiometer and spectrometer techniques and technologies. Langley researchers were the first to demonstrate a solar occultation sensor in space and provided the first measurements of tropospheric trace gas from space using gas filter correlation radiometry. The second primary technology product area in the Langley R\&T program is structures and materials. Much of the current effort here is focused on the problems associated with large apertures where the objective is to accommodate high-resolution spacebased sensing systems in smaller, reduced-costs launch packages. This challenge draws on Langley expertise in deployable and inflatable structures, breakthrough materials, structural dynamics and geometry control, and lightweight multifunctional structures.

Research and technology development in both the instruments and sensors and structures and materials product areas encompasses all levels of technology maturation including conceptual development and analysis, component development and demonstration, system/subsystem development and demonstration, and protoflight space validation. The following sections provide selected examples of current activities at Langley across the various levels of technology maturity.

\section{LIDAR TECHNOLOGY DEVELOPMENT}

There are considerable technological challenges associated with enabling lidar atmospheric measurements from space. Principle among those is obtaining adequate signal-to-noise measurements within a compact, affordable system. To that end, 
technology developments at LaRC are focused on highenergy, high-efficiency, conductively-cooled laser transmitters, precision deployable telescopes, and highly efficiency detectors. Since the operating wavelengths vary according to the desired absorption line of the atmospheric species to be measured (water vapor, ozone, carbon dioxide, etc.); it is necessary to literally invent new solid state laser sources operating from the ultraviolet to the infrared. To assist in that effort, Langley technologists have developed unique quantum mechanical modeling tools that allow laser performance of solid state crystals to be predicted in advance of committing to hardware development. As an example, quantum mechanical modeling accurately predicted the performance (wavelength, efficiency, etc.) of the solid state material (holmium and thulium doped YLF) which was then used to develop an eye-safe, 2-micron laser for a coherent winds lidar.

After determining the appropriate characteristics of the solid state material via modeling and spectroscopy, LaRC technologists, working with industry partners, develop a breadboard laser for laboratory testing. The laser is then characterized in the laboratory in terms of pulse format, energy per pulse (typically 0.5 to $1.0 \mathrm{~J}$ ), beam quality, laser line-width, and efficiency. Initially, for cost considerations, the laser is pumped via a flash lamp or another laser. However, to achieve the desired efficiency, it must eventually be tested in a diode pumped configuration (figure 2). These diode arrays are developed to LaRC specifications often by small, innovative businesses. Langley researchers are currently investigating new pump configurations (e.g., end pumped disks) in an effort to improve overall transmitter efficiency. After successful component and system testing the lab, the laser transmitter development is at the appropriate stage for entering the Instrument Incubator Program where more advanced field and aircraft demonstrations can be performed.

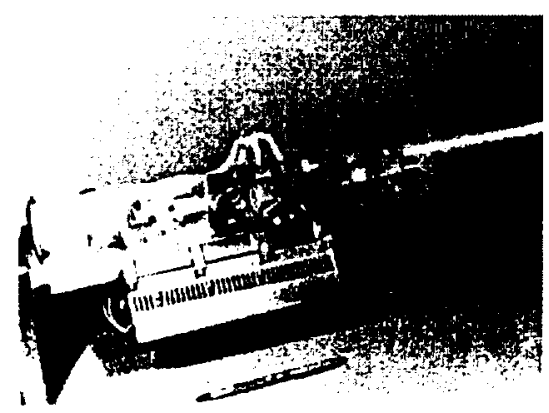

Figure 2. Diode-pumped laser transmitter.
High output energy from the latser source is one method for increasing lidar return signal. Another is to increase the size of the receiver aperture. Research is currently underway at Langley to develop critical components for a 3-meter class, precision deployable telescope. The deployable feature enahles the telescope pre-deployment launch palikage to be accommodated in a small, less costly launch vehicle. Researchers at LaRC have developed precision latch and hinge mechanisms (figure 3) which feature highly linear operation with minimum hysterisis to enable a deployment accuracy of 2 microns.

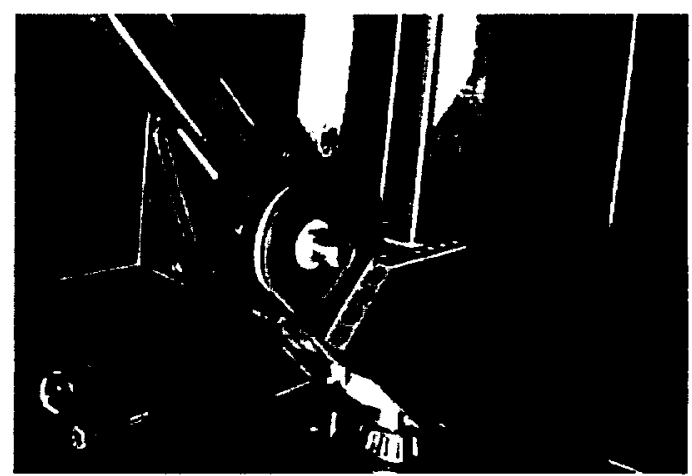

Figure 3. Precision latch and hinge deployment mechanisms.

In addition, lightweight composite mirror panels have been developed under an SBIR with Composite Optics Inc. (figure 4) with an areal density of $100 \mathrm{~kg}$ per square meter. Currently, the University of Colorado is performing dynamics testing and deployment accuracy and repeatability tests with an integrated panel/mechanisms assembly.

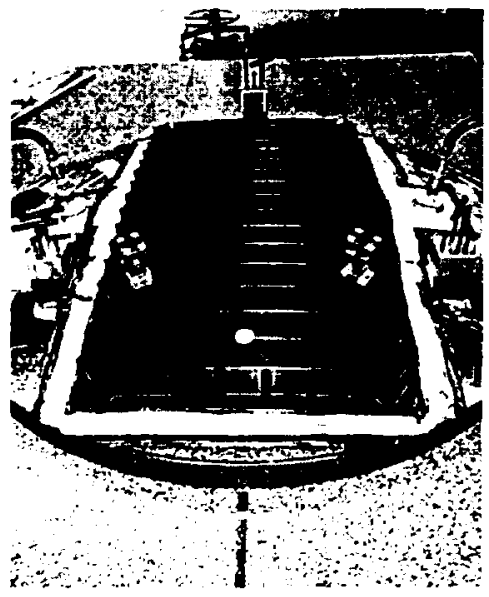

Figure 4. Composite mirror panels. 
One major driver for such a "photon collecting" telescope would be an ultraviolet differential absorption lidar for space-based measurements of tropospheric ozone.

\section{GAS AND AEROSOL MONITORING SENSORCRAFT(GAMS)}

Langley pioneered the atmospheric measurement technique known as solar occultation dating back to the first such measurement in space during the Apollo Soyuz mission. Since solar occultation images the sun through the Earth's atmosphere, the technique is selfcalibrating offering long-term stability. It also provides high-vertical resolution measurements of the stratosphere and upper troposphere. SAGE III is the latest solar occultation instrument being developed to measure ozone, aerosols, clouds, water vapor, and other species. Multiple copies of SAGE III are under development for launch on a Russian Meteor spacecraft and as a Space Station attached payload. The weakness of this technique is coverage since there is only 2 measurement opportunities per orbit. It has been determined that 6 SAGE III instruments would have to be placed in the appropriate orbit to achieve weekly coverage of the Earth. Obviously, this fleet approach is affordable only if the launch costs, instrument/ spacecraft per copy development cost, and fleet operating cost can be kept to a minimum. The approach being pursued at Langley is to utilize advanced instrument and spacecraft technologies to develop compact, highly integrated "sensorcraft" where the boundary between the spacecraft bus and instrument become less distinct. Essentially, the sensorcraft concept integrates the resource requirements of both to minimize size, power consumption and cost. A fleet of intelligent sensorcraft constitutes the next generation of SAGE measurement capability and is referred to as Gas and Aerosol Monitoring Sensorcraft or GAMS (figure 5).

The science mission envisioned for GAMS would provide multi-wavelength aerosol extinction and full $\mathrm{O}_{3}, \mathrm{O}_{2}$, and $\mathrm{H}_{2} \mathrm{O}$ profile coverage over a spectral range of $430 \mathrm{~nm}$ to $1020 \mathrm{~nm}$. GAMS concept studies project the sensorcraft volume to be about 0.1 cubic meters with a mass of $50 \mathrm{Kg}$ and power of 50 watts. Real time, onboard data processing will be performed to produce EOS Level-2 science data at rate which can be down linked directly to a user's workstation over a commercial LEO communications system. Studies indicate that a GAMS fleet of 6 would offer a $65 \%$ cost savings compared to the more traditional approach where SAGE III instruments would be mated with conventional spacecraft busses.

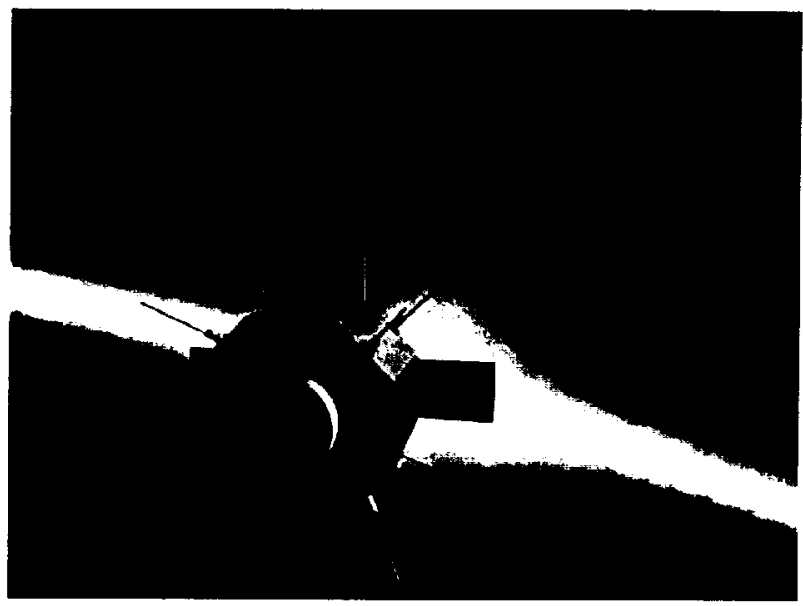

Figure 5. Gas and Aerosol Monitoring Sensorcraft (GAMS).

After a successful 24-month component technology development effort at LaRC, the GAMS concept was ready to enter the system/subsystem demonstration phase. In 1998, a GAMS proposal was selected by the ESE through an Instrument Incubator Program (IIP) competitive process. Consistent with the scope of the IIP, this effort focuses on the development and demonstration of those functions specific to the science instrument and flight data processing hardware and software. In this particular case, it just so happens that those functions had been judged to be the technical "tall poles" at an earlier design review.

The IIP/GAMS project will develop and test a novel two-axis gimbal system for spectrometer scan control and will integrate and test the grating spectrometer and Digital Signal Processing (DSP) hardware and software subsystems. The integrated spectrometer/DSP is currently undergoing functional/performance laboratory and roof top testing. Late in the year, a series of sunrise/sunset flight field demonstrations will be conducted onboard NASA LaRC's 757 at 42,000 feet. Several advanced technologies will be validated via the IIP/GAMS field demonstrations. In addition to the DSP for onboard data processing, these include a high-resolution, deepwell, CCD linear array, an advanced piezo-electric motor drive for the two-axis gimbal, and a carboncarbon spectrometer head.

Since the sensorcraft fleet approach to atmospheric sensing is a radical departure from convention, it is 
likely to require actual validation in space before being accepted by the larger science community. Therefore, subsequent to a successful IIP demonstration, the development and operation of a tleet of 2 or more fullup, Gas and Aerosol Monitoring Sensorcraft would be an ideal flight experiment for the New Millennium Program (NMP).

\section{GEOSTATIONARY IMAGING FTS (GIFTS)}

NASA Langley Research Center (LaRC), has teamed with University of Wisconsin-Madison, Space Dynamics Laboratory (SDL) of Utah State University and National Oceanographic and Atmospheric Administration (NOAA) to develop a new measurement concept known as Geostationary Imaging Fourier Transform Spectrometer (GIFTS). GIFTS was recently selected by the New Millennium Program for development and space validation with launch scheduled in 2004 (figure 6). The imaging FTS is an innovative measurement technique for water vapor, temperature, and wind sounding that will provide critical weather information not achievable through any existing space-based observatory. It is also capable of measuring the motion of clouds and transport pollutants in the atmosphere.

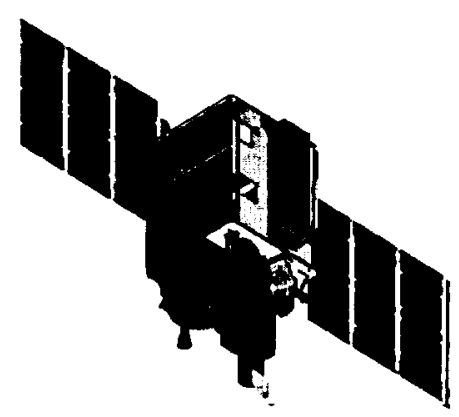

Figure 6. GIFTS instrument on a Geostationary satellite.

In addition to the demonstrating an advanced measurement technique, several technologies will be flight tested and validated in the NMP GIFTS mission. These include 2-D imaging arrays, active cooling, fast digital signal processing, pointing and control, radiation protection, and lightweight materials. GIFTS represents a first step toward incorporating technological breakthroughs into the next generation of operational weather observing systems.

\section{ENABLING THE EARTH SCIENCE VISION}

The Earth Science Enterprise, through its Vision Initiative (ESVI), looks to understand the Earth System and then go beyond understanding to the benefits derived from prediction of the Earth System. Proactive environmental prediction would enable accurate twoweek weather forecasting, accurate climate prediction, and prediction of natural hazards as examples. The ESE seeks to develop an architecture that connects and focuses science, measurements, and technology investments toward realizing the benefits of these applications in the future.

The R\&T program at Langley is currently pursuing the reach goals established by the ESVI within four major technology themes: advanced sensors, sensorwebs, information synthesis, and access to knowledge. Under the advanced sensors theme, Langley is developing advanced tunable lidar technologies described in a previous section of this paper. In addition, the structures and materials expertise at LaRC is being applied to the challenges of developing large, ultra-lightweight, inflatable telescopes and antennas for high-resolution imaging and microwave radiometry.

The sensorweb concept incorporates multiple, reprogammable/reconfigurable, observational systems operating collaboratively from multiple vantage points (LEO, GEO, L1, etc.) to provide a robust and adaptive earth observing network. However, for this concept to be feasible, observational nodes on the sensorweb must be low cost, autonomous, and intelligent, micro/nanosatellites. Developments to date at Langley in the GAMS R\&T project are intended to enable a "smart" sensorcraft capability for Earth Science. Therefore, GAMS can be viewed as an initial step toward realizing the sensorweb concept. A logical next step would be a NMP space flight experiment featuring multiple, collaborative sensorcraft at various orbital locations.

The EOS Distributive Active Archive Center (DAAC) for tropospheric chemistry, radiation budget, clouds and aerosol data resides at Langley Research Center. Consistent with capabilities identified by the ESVI for access to knowledge, the DAAC has developed a system called the Langley TRMM Information System (LaTIS). Specifically, this system deals with high volume satellite data production, 
archive, and distribution. To support early data fusion work, a facility known as Cave Automated Virtual Environment (CAVE) has been built to support research into how to benefit from immersive environments for 3-dimensional visualization of geospatial data sets.

\section{SUMMARY}

NASA Langley has over 20 years of experience in spaceflight, airborne, and ground-based scientific measurements. These measurements have contributed significantly to our understanding of the Earth's atmosphere. LaRC possesses a unique end-to-end capability to go from the scientific question to mission development to data analysis. The cornerstone of this capability is a robust research and technology program which spans the technology readiness levels from concept to component development to system prototypes to flight validation. The strength of the Langley R\&T approach is an integrated team of scientists, engineers, and technologists working together to achieve science-driven, technological innovation. The focus is on reducing cost and enabling new measurements for the next generation EOS missions and the realization of the Earth Science vision. 